\title{
Validação da Avaliação Terapêutica Ocupacional (ATO) para Adolescentes e Adultos com Deficiência Visual por painel de especialistas $^{1}$
}

\author{
Validación de la Evaluación Terapéutica Ocupacional (ATO) para \\ Adolescentes y Adultos con Discapacidad Visual por panel de expertos \\ Validation by panel of experts of the Occupational Therapy Assessment \\ (ATO) of Adolescents and Adults with Visual Impairment
}

\author{
Marissa Romano da Silva \\ Rita de Cássia letto Montilha ${ }^{3}$
}

Recibido: Recibido: 1 de junio 2020 Enviado para modificación: 12 de agosto $2020 \bullet$ Aceptado: 22 de agosto 2020 Da Silva, M.R. y Montilha, R.C.I. (2020). Validação da Avaliação Terapêutica Ocupacional (ATO) para Adolescentes e Adultos com Deficiência Visual por painel de especialistas. Revista Ocupación Humana, 20 (1), 27- 48. https://doi. org/10.25214/25907816.947

\section{RESUMO}

Descreve-se o processo de validação da Avaliação Terapêutica Ocupacional (ATO) para Adolescentes e Adultos com Deficiência Visual, através de juízes especialistas. Pesquisa com delineamento misto, com estratégia qualitativa exploratória descritiva e quantitativa descritiva, para compor a análise dos dados. Foi organizado um painel de especialistas para utilização da ferramenta técnica Delphi. O processo de coleta de dados ocorreu por meio

\footnotetext{
1 O presente manuscrito é parte integrante da pesquisa de doutorado desenvolvida por Marissa Romano da Silva no Programa de Pós-Graduação em Saúde, Interdisciplinaridade e Reabilitação da Faculdade de Ciências Médicas da Universidade Estadual de Campinas - UNICAMP, intitulada "Validação da Avaliação Terapêutica Ocupacional (ATO) para Adolescentes e Adultos com Deficiência Visual". A pesquisa não recebeu financiamento.

${ }^{2}$ Terapeuta ocupacional. Mestre em Saúde, Interdisciplinaridade e Reabilitação. Doutoranda em Saúde, Interdisciplinaridade e Reabilitação, Universidade Estadual de Campinas. Campinas, Brasil. marissaromano.to@gmail.com iD https://orcid.org/0000-0003-3661-0532

${ }^{3}$ Terapeuta ocupacional. Mestra em Neurociência. Doutora em Ciências Médicas. Professora, Universidade Estadual de Campinas. Campinas, Brasil. rcietto@fcm.unicamp.br. iD https://orcid. org/0000-0003-3741-0006
} 
eletrônico, para envio e recepção dos documentos. A validade é estabelecida quando houver consenso dos especialistas, que inclui estabilidade nas sugestões e índice de validade de conteúdo total igual ou maior que $90 \%$ (IVC total $\geq 0,90$ ). Oito profissionais da saúde participaram das três rodadas da técnica. Cada etapa apresentou IVC próprio, recebeu sugestões e comentários que possibilitaram modificações, adições de questões pertinentes e melhoramentos na estrutura, conteúdo e organização da Avaliação. Na última rodada, foi estabelecido o consenso com IVC = 0,97 (97,91\%) e ausência de sugestões que provocassem alterações na Avaliação. Segundo os juízes especialistas, a Avaliação é válida, pois avalia o que se propõe. Possui linguagem acessível, estrutura e conteúdo relevantes, claros, adequados para atingir seu objetivo máximo: avaliar a funcionalidade de adolescentes e adultos com deficiência visual, por meio de métodos de entrevista e observação direta.

\title{
PALAVRAS-CHAVE
}

Terapia Ocupacional, pessoas com deficiência visual, Classificação Internacional de Funcionalidade, Incapacidade e Saúde, estudo de validação, técnica Delfos

\section{RESUMEN}

Se describe el proceso de validación de la Evaluación Terapéutica Ocupacional (ATO) para Adolescentes y Adultos con Discapacidades Visuales, a través de expertos. Se realizó una investigación con diseño mixto, estrategia exploratoria cualitativa y análisis de datos cuantitativo descriptivo. Se organizó un panel de expertos, utilizando la técnica Delphi. Los datos se recopilaron electrónicamente. La validez se estableció por consenso de expertos, incluyendo la estabilidad en las sugerencias y un índice de validez de contenido total mayor o igual al $90 \%$ (IVC total $\geq 0.90$ ). Participaron ocho profesionales de la salud en las tres rondas de la técnica. En cada etapa se calculó el IVC y se recibieron sugerencias y comentarios que permitieron modificar, agregar preguntas relevantes y mejorar la estructura, el contenido y la organización de la Evaluación. En la última ronda se llegó a consenso, con IVC $=0.97(97.91 \%)$ y ausencia de nuevas sugerencias de cambio. Según los expertos, la Evaluación es válida, evalúa lo que se propone; tiene un lenguaje accesible, una estructura y contenido relevantes; es clara, objetiva y adecuada para alcanzar su principal objetivo: evaluar la funcionalidad de adolescentes y adultos con discapacidad visual a través de entrevista y observación directa.

\section{PALABRAS CLAVE}

Terapia Ocupacional, personas con deficiencia visual, Clasificación Internacional del Funcionamiento, de la Discapacidad y de la Salud, estudio de validación, técnica Delfos

\begin{abstract}
This paper describes the validation process of the Occupational Therapy Assessment (ATO) for Adolescents and Adults with Visual Impairment, through expert judges. A research with mixed method, an exploratory qualitative strategy and descriptive quantitative data analysis was carried out. A panel of experts was set up using the Delphi technique tool. Data were collected electronically. The validity was established upon experts' consensus, which included stability in the suggestions and total Content Validity Index (S-CVI/Ave) $\geq 0.90$ (90\%). Eight health professionals participated in the three rounds of the technique. The CVI was calculated on each stage, and suggestions and comments were received, which made
\end{abstract}


it possible to modify, add relevant questions and improve the structure, content and organization of the Assessment. A consensus was established in the last round, with S-CVI/Ave $=0.97(97.91 \%)$ and absence of suggestions that would cause changes in the Assessment. According to the expert judges, the Assessment is valid, evaluates what is proposed and is written in accessible language. It also has a relevant structure and contents; it is clear, objective, and adequate to achieve its primary objective: to evaluate the functionality of visually impaired adolescents and adults, through interview methods and direct observation.

\section{KEYWORDS}

Occupational Therapy, visually impaired persons, International Classification of Functioning, Disability and Health, validation study, Delphi technique

\section{Introdução}

A Terapia Ocupacional tem seu conhecimento diretamente influenciado por diversas ciências, tais como as ciências biológicas, psicológicas e sociais. Tal influência se dá pelo contexto e pelo momento histórico em que está inserida. Sendo influenciada por diversos aspectos, a profissão, em seu processo de produção de conhecimento e intervenção, foi e vem sendo construída com seus métodos em constante movimento (Medeiros, 2010). Os caminhos trilhados no decorrer de sua formação e concretização, enquanto profissão, fazem com que não apresente uma história linear, refletindo sua expansão gradual para diversos campos de atuação (De Carlo \& Bartalotti, 2001; Medeiros, 2010). Independente do campo, seu objeto de estudo é a ocupação humana (Medeiros, 2010). A ocupação assume importâncias, funções e significados diferentes na vida das pessoas, envolve o conhecer, o fazer, o aprender, o habituar e compõe, até mesmo, a identidade humana (Dickie, 2011).

A articulação entre as ciências e o contexto social, político, econômico e cultural reflete a maneira como a Te- rapia Ocupacional compreende o ser humano. Como ela o reconhece em constante construção, transformação e sob influência do contexto (Medeiros, 2010). Considera, em seu processo avaliativo, os contextos e ambientes da pessoa, incluindo o cultural, pessoal, físico, social, temporal e virtual (American Occupational Therapy Association - AOTA, 2015). A atenção sobre o ambiente como influenciador, com papel transformador, é intrínseca à profissão, compondo sua avaliação e atuação à necessidade de conhecer quais os fatores ambientais têm papel fundamental no desempenho do indivíduo.

No Brasil, no cotidiano do terapeuta ocupacional, é comum a realização de avaliações organizadas pelo profissional para sua própria prática ou mesmo a utilização de protocolos institucionais não validados. Além disso, utilizam avaliações padronizadas e validadas, em sua maioria advindas do exterior, tendo passado por processos de tradução, adaptação transcultural e validação para o público nacional. Entretanto, há pouco material de avaliações terapêuticas 
ocupacionais construídas, validadas e publicadas por terapeutas brasileiros para o público brasileiro. No Brasil, de forma geral, a profissão não tem a tradição de construir instrumentos de avaliação terapêutico ocupacionais e muito desse movimento pode ser atribuído à sua trajetória no país. Algumas problematizações podem ser feitas quanto aos possíveis motivos que podem levar os terapeutas ocupacionais brasileiros à pouca construção e adesão ao uso sistemático de avaliações padronizadas na prática clínica.

A Terapia Ocupacional foi se construindo a partir da medicina e, desta maneira, influenciada pela divisão em áreas de especialidades, fragmentando conhecimentos e práticas clínicas. As ações eram desenvolvidas por enfermeiros e assistentes sociais, treinados por médicos acerca do uso de atividades como tratamento. No Brasil, a prática com uso de atividades teve seu início na reabilitação física, na época, ainda considerada uma profissão paramédica e dependente das prescrições médicas para desenvolver-se. Ao final da década de 1940, a cobrança pela sistematização do conhecimento terapêutico ocupacional, pelo pragmatismo e mensuração das intervenções, levou a um perfil da profissão cada vez mais especializado, em busca de um status científico para suas atuações (De Carlo \& Bartalotti, 2001; Medeiros, 2010). De acordo com Medeiros (2010), "A Terapia Ocupacional estava sendo, então, a terapia das especialidades médicas (...)." (p.111); esse momento é chamado de reducionismo.
Em 1970, nos Estados Unidos, discussões e reflexões em relação aos conceitos que envolvem saúde-doença, corpo, atividade, ocupação e a relação de submissão à área médica, tomaram corpo. Movimentos críticos ao modelo mecanicista do reducionismo, que não considerava as necessidades do sujeito e seu envolvimento nas situações de vida, foram iniciados (Medeiros, 2010).

Como resultado desse período de questionamentos foi desenvolvido o chamado Modelo da Ocupação Humana, por Kielhofner e Burke, que teve forte influência na prática da profissão, principalmente, nos Estados Unidos. Entretanto, no Brasil, o crescimento e fortalecimento da Terapia Ocupacional não foi seguido pelo desenvolvimento e adoção de modelos de prática ${ }^{4}$, o que pode ser atribuído ao contexto político e histórico da época (Cruz, 2018).

Em determinado momento da história da profissão no Brasil, houve uma divisão entre os profissionais que se mantiveram guiados pelo movimento e abordagens da ocupação; e outros que partiram para reflexão do fazer da Terapia Ocupacional, à luz de referenciais da filosofia, educação, sociologia e antropologia. Estes últimos, críticos ao Modelo da Ocupação Humana, compreendem esse modelo distante das problemáticas da sociedade e ainda próximo ao paradigma reducionista (Cruz, 2018).

Partindo dessa breve exposição e compreendendo que rumos tomados há décadas, inevitavelmente, ecoam

\footnotetext{
${ }^{4}$ Embora não tenhamos pesquisas divulgadas no Brasil sobre quais os modelos são seguidos pelos terapeutas ocupacionais brasileiros (Cruz, 2018).
} 
nos dias atuais, deixamos, aqui, alguns questionamentos e problematizações. As críticas aos modelos de base positivista que, de alguma forma, inserem a avaliação padronizada como parte fundamental do processo em Terapia Ocupacional, pode ter afastado a profissão no Brasil do desenvolvimento de instrumentos? Isso reflete a escassez de instrumentos brasileiros? Em que momento da Terapia Ocupacional brasileira estamos agora? Há resistência do uso de instrumentos de avaliação em nossa prática brasileira?

Ainda se faz necessário procurar por respostas ou, ao menos, sinais que indiquem os caminhos a esses questionamentos. Porém, acreditamos ser possível uma prática contextualizada e ampliada, sem negar a sistematização das intervenções terapêuticas ocupacionais.

A Avaliação Terapêutica Ocupacional (ATO) para Adolescentes e Adultos com Deficiência Visual (Silva, 2016), aqui validada, busca estabelecer um diálogo cuidadoso e respeitoso ${ }^{5}$ entre o saber sistemático - através do uso de qualificadores, que refletem objetivamente a magnitude da deficiência e das dificuldades - e a avaliação e atuação, com vistas à complexidade do indivíduo, as subjetividades e interesses próprios.

A Avaliação faz uso da entrevista semiestruturada para a identificação do desempenho ocupacional, influenciadores cotidianos, interesses, prioridades, expectativas. Assim como, a realização de tarefas funcionais para observação das capacidades e sua posterior qualificação na Classificação Internacional de Funcionalidade, Incapacidade e Saúde - CIF (Organização Mundial da Saúde - OMS, 2015).

Na revisão bibliográfica, desenvolvida durante o processo de construção da Avaliação Terapêutica Ocupacional (ATO) para Adolescentes e Adultos com Deficiência Visual (Silva, 2016), não foram identificadas avaliações - padronizadas, validadas e publicadas -, desenvolvidas para terapeutas ocupacionais, que tivessem como público alvo pessoas com deficiência visual e que utilizassem como base conceitual a Classificação CIF. A Classificação considera a forte influência do contexto sobre o indivíduo, de forma positiva ou negativa. Entende a relação das condições de saúde e do ambiente como uma interação dinâmica, passível de gerar mudanças no estado de saúde (OMS, 2015).

A escassez de avaliações terapêutico ocupacionais e, especificamente, dentro da área da deficiência visual, utilizando as concepções e estrutura da Classificação Internacional de Funcionalidade, Incapacidade e Saúde - CIF, justificou a construção de um instrumento que realizasse um rastreio funcional da pessoa com deficiência visual. Tal instrumento identifica os principais aspectos que influenciam sua vida plena, engajamento, participação e qualidade de vida. A descrição do processo de construção da Avaliação Terapêutica

\footnotetext{
${ }^{5} \mathrm{O}$ termo "respeitoso" é empregado na medida em que a história da Terapia Ocupacional não pode ser negada, independente dos paradigmas e modelos vivenciados durante seu processo de construção até a contemporaneidade. Toda sua história deve ser respeitada, todo o percurso deve ser valorizado. As críticas devem ser feitas para que novos passos sejam dados. Dessa forma, estabelecemos esse diálogo com respeito aos caminhos que trilhamos no passado, aos que estamos trilhando e aos que iremos trilhar.
} 
Ocupacional (ATO) para Adolescentes e Adultos com Deficiência Visual é relatada em Silva (2016).

Contudo, instrumentos de avaliação, para serem amplamente utilizados, devem passar por processos que garantam que meçam o que se propõem medir e que possuam conteúdo adequado e suficiente para identificação das informações necessárias de determinado constructo. Um processo através do qual uma avaliação pode ser testada é a validade, com significado e métodos próprios.

A validade diz respeito ao instrumento medir o que se propõe e pode ser estabelecida através de algumas estratégias (Lynn, 1986; Almeida, 2003; Martins, 2006; Alexandre \& Coluci, 2011; Souza, Alexandre \& Guirardello, 2017), dentre elas, a validade de face (ou aparente) e a validade de conteúdo. A validade de face (ou aparente) está relacionada à evidência aparente da integridade da avaliação; se o instrumento faz sentido ao campo ou área que se propõe avaliar. É avaliada por um painel de especialistas. Todo instrumento de avaliação deve garantir sua validação de face.

A validade de conteúdo deve garantir que os principais aspectos, relacionados àquele conteúdo, sejam incluídos na avaliação. Pode ser obtida através do questionamento à pessoa/ população, ao grupo, ou a um painel de especialistas acerca da clareza, objetividade, abrangência dos itens e propriedade do instrumento.

Uma estratégia que pode ser utilizada para garantir a validade de um instrumento de avaliação, através da consulta a especialistas, é a técnica Delphi
(Faro, 1997; Coutinho et al., 2013). O objetivo deste manuscrito é descrever o processo de validação de face e conteúdo da Avaliação Terapêutica Ocupacional (ATO) para Adolescentes e Adultos com Deficiência Visual, através de um painel de especialistas.

A Avaliação Terapêutica Ocupacional (ATO) para Adolescentes e Adultos com Deficiência Visual foi construída como pesquisa de mestrado no Programa de Pós-Graduação em Saúde, Interdisciplinaridade e Reabilitação, da Faculdade de Ciências Médicas da Universidade Estadual de Campinas, no período de 2014 a 2016 (Silva, 2016). A validação da Avaliação é resultado da pesquisa de doutorado realizada no referido Programa e desenvolvida entre os anos de 2016 e 2020 (Silva, 2020).

\section{Metodologia}

Pesquisa com delineamento misto, através da estratégia qualitativa exploratória descritiva, compondo com o procedimento quantitativo descritivo para análise dos dados (Gil, 2008).

Para o processo de validação de face e de conteúdo, optou-se por utilizar, exclusivamente, o meio eletrônico como mecanismo de envio e recepção dos documentos durante o processo de validação. Ao utilizar o mecanismo eletrônico, não foi necessário um local de encontro entre pesquisadora principal e participantes.

Para o processo de validação, foi composto um painel de especialistas, em conformidade à estratégia de coleta de dados escolhida, a técnica Delphi, que será, brevemente, descrita poste- 
riormente. Foi constituída uma amostra não probabilística, por acessibilidade ou conveniência (Gil, 2008). Os juízes especialistas desta pesquisa compreenderam profissionais da área da saúde (envolvidos em pesquisa e/ou ensino e/ou prática clínica). Tais profissionais eram atuantes em deficiência visual - e/ ou com conhecimento no processo de construção e validação de instrumentos de avaliação; e/ou conhecedores da Classificação Internacional de Funcionalidade, Incapacidade e Saúde -CIF; e/ou terapeutas ocupacionais. Foram excluídos profissionais que estivessem envolvidos, em qualquer momento, na construção da Avaliação, bem como aqueles com qualquer conflito de interesse com a pesquisa, e/ou não aceitassem/assinassem o Termo de Consentimento Livre e Esclarecido.

Para a formação de um painel de especialistas, o número de participantes ainda não é consenso na literatura (Faro, 1997; Grant \& Davis, 1997; Powell, 2003; Habibi et al, 2014; Waggoner et al., 2016; Marques \& Freitas, 2018). Segundo Lynn (1986), para se formar um painel de especialistas, faz-se necessário o mínimo de cinco sujeitos participantes. O número máximo não fora estabelecido, entretanto, sugere-se que não seja excedida a participação de mais de dez especialistas. Foram convidados a participar treze profissionais. Oito especialistas aceitaram, assinaram o Termo de Consentimento Livre e Esclarecido e participaram durante todo o processo de validação.

Para que fossem utilizadas estratégias de validação de conteúdo e de face, através do suporte de juízes especialistas, optou-se pela utilização da técnica Delphi, que se constitui como uma ferramenta apropriada para estabelecer a validade de instrumentos de avaliação (Faro, 1997; Coutinho et al., 2013). A técnica Delphi é uma estratégia que permite a obtenção de informações sobre determinado assunto, a partir da construção participativa de especialistas (Faro, 1997; Almeida, 2003; Almeida, 2004; Almeida et al., 2008; Coutinho et al., 2013). Juízes especialistas são convidados a julgar determinados assuntos por meio de seu questionamento, possibilitando que expressem suas opiniões.

Através da compilação das opiniões dos especialistas acerca de: conteúdo, apresentação, propriedade, clareza, originalidade, organização, linguagem, itens necessários, porém ausentes, itens desnecessários, comentários e sugestões de um instrumento; é possível considerar a avaliação validada (Faro, 1997; Almeida, 2003; Almeida, 2004; Almeida et al., 2008; Coutinho et al., 2013).

A sequência de compilar resultados, rever e reconfigurar o questionário, redistribuir aos juízes especialistas e analisar os novos resultados deve haver até o alcance do consenso. Para a técnica, consenso diz respeito ao momento em que as respostas forem estáveis, sem novas sugestões e opiniões que levem a mudanças na avaliação, e quando houver o alcance do índice de valide de conteúdo (IVC) por item e total estabelecidos pela literatura (Lynn, 1986; Faro, 1997; Almeida, 2004; Polit \& Beck, 2006; Almeida et al., 2008; Revorêdo et al., 2015; Marques \& Freitas, 2018). A literatura aponta a realização de duas a quatro rodadas para o alcance do consenso (Kayo \& Securato, 1997; Powell, 2003; Almeida et al., 2009; Waggoner et al., 2016; Massaroli et al., 2017; Marques \& Freitas, 2018). 
Nesta pesquisa, inicialmente, foram enviados, por correio eletrônico, os convites para profissionais da saúde que corresponderam aos critérios da pesquisa, juntamente com o Termo de Consentimento Livre e Esclarecido. Após a devolutiva de todos os termos de consentimento, devidamente assinados, em prazo previamente estipulado, a Avaliação Terapêutica Ocupacional (ATO) para Adolescentes e Adultos com Deficiência Visual e a $1^{\text {a }}$ rodada de questionário foram enviados, na mesma data, para todos os participantes. O participante tinha o acesso ao questionário através de um link que o direcionava à plataforma do Google Forms. As rodadas seguintes também foram distribuídas por meio eletrônico e seguiram com a mesma lógica de envio da Avaliação modificada e com o link do novo questionário.

Estratégias descritivas, qualitativas e quantitativas, foram utilizadas para a análise dos dados. Pesquisas que fazem uso da técnica Delphi têm os procedimentos de análise transcorrendo na coleta, haja vista que a rodada seguinte inicia após a análise dos dados do questionário anterior (Massaroli et al., 2017).

Para o tratamento dos comentários, sugestões e opiniões, foi realizada a categorização dos aspectos identificados, seguida pela análise aprofundada de cada item à luz do referencial teórico e a posterior inserção ou retirada do item correspondente na Avaliação.

Para a análise quantitativa, através do estabelecimento da concordância entre os juízes especialistas, foram calculados os índices de validade de conteúdo por item (IVC item) e total (IVC total). Para calcular o índice de validade de conteúdo por item foi utilizada a fórmula IVC item $=\mathrm{n}^{\circ}$ de respostas positivas $/ \mathrm{n}^{\circ}$ total de respostas. $\mathrm{O}$ índice de validade de conteúdo total pode ser obtido por meio do cálculo IVC total = $\mathrm{n}^{\mathrm{o}}$ de respostas positivas/ ( $\mathrm{n}^{\mathrm{o}}$ juízes $\mathrm{X} \mathrm{n}^{\mathrm{o}}$ itens) (Polit \& Beck, 2006).

Em conformidade com a literatura (Lynn, 1986; Polit \& Beck, 2006), a presente pesquisa considerou o IVC total padrão $\geq 0,90(90 \%)$ e o IVC item padrão $\geq 0,78(78 \%)$. Questões com IVC item $\leq 0,75(75 \%)$, de concordância entre os juízes especialistas, foram compulsoriamente revistas; e os itens que correspondem à Avaliação, obrigatoriamente modificados ou excluídos (Lynn, 1986). Questões com índice de validade de conteúdo, item compreendido no intervalo entre 0,75 $(75 \%)$ e $0,78(78 \%)$, foram revistas, entretanto, não compulsoriamente excluídas ou alteradas.

\section{Aspectos éticos}

Esta pesquisa foi aprovada pelo Comitê de Ética em Pesquisa da Faculdade de Ciências Médicas, da Universidade Estadual de Campinas, sob o parecer $\mathrm{n}^{\circ}$ 2.167.526/17.

\section{Resultados e discussão}

A tabela 1 apresenta uma síntese do processo realizado, através da implementação da técnica Delphi, com seus resultados de índice de validade de conteúdo total apresentados. Cada rodada, com seus questionários e resultados próprios, será melhor descrita nos tópicos a seguir.

Cada etapa da pesquisa de validação 
contemplou a distribuição de questionário, a recepção dos pareceres dos participantes, a compilação e análise dos dados, bem como a modificação e adaptação da Avaliação Terapêutica Ocupacional. Contou também com a consecutiva construção de um novo questionário, enviado ao painel de juízes especialistas, para nova apreciação.

O processo de validação, do envio dos convites à análise da terceira rodada, teve duração de doze meses. O consenso, qualitativo e quantitativo, foi

Tabela 1. Síntese das três rodadas do processo de validação - técnica Delphi.

\begin{tabular}{|c|c|c|}
\hline \multicolumn{2}{|r|}{ Rodada da técnica Delphi } & Resultado \\
\hline $1^{\mathrm{a}}$ rodada & $\begin{array}{l}\text { Envio aos juízes especialistas da } \mathrm{ATO}_{1} \\
\text { - versão inicial. } \\
\text { Os juízes deveriam julgar: clareza, } \\
\text { objetividade, conteúdo, linguagem, } \\
\text { estrutura. }\end{array}$ & $\begin{array}{l}\text { Caracterização do painel de juízes } \\
\text { especialistas. } \\
\text { Adequação da } \text { ATO }_{1} \text { resultando na } \\
\mathrm{ATO}_{2} \text { e construção de um manual } \\
\text { para sua aplicação. } \\
\text { IVC total }=0,892(89,2 \%)\end{array}$ \\
\hline $2^{\mathrm{a}}$ rodada & $\begin{array}{l}\text { Envio da } \mathrm{ATO}_{2} \text { (Avaliação e manual) } \\
\text { aos juízes especialistas - julgamento } \\
\text { das adequações anteriores. }\end{array}$ & $\begin{array}{l}\text { Adequação da } \mathrm{ATO}_{2} \text { e seu respectivo } \\
\text { manual resultando na } \mathrm{ATO}_{3} \text { e } \\
\text { adequação do manual. } \\
\text { IVC total }=0,941(94,1 \%)\end{array}$ \\
\hline $3^{a}$ rodada & $\begin{array}{l}\text { Envio da } \mathrm{ATO}_{3} \text { - julgamento das } \\
\text { alterações realizadas após o resultado } \\
\text { da 2a etapa da técnica Delphi. } \\
\text { Considerações, observações e } \\
\text { sugestões gerais quanto ao } \\
\text { instrumento em validação. }\end{array}$ & $\begin{array}{l}\text { Estabelecido consenso. } \\
\text { Versão final validada da ATO e seu } \\
\text { manual. } \\
\text { IVC total }=0,979(97,9 \%)\end{array}$ \\
\hline
\end{tabular}

Fonte: Silva (2020)

estabelecido na terceira rodada da técnica Delphi, o que está em conformidade com a literatura de referência - que aponta a realização de duas a quatro rodadas para obtenção de consenso (Kayo \& Securato, 1997; Powell, 2003; Almeida et al., 2009; Waggoner et al., 2016; Massaroli et al., 2017; Marques \& Freitas, 2018).

\section{$1^{a}$ rodada da técnica Delphi}

A $1^{\text {a }}$ etapa do processo de validação, através da técnica Delphi, compreendeu o envio da avaliação construída em Silva (2016): Avaliação Terapêutica Ocupacional para Adolescentes e Adultos com Deficiência Visual (aqui chamada de $\mathrm{ATO}_{1}$ ). 
A submissão do primeiro questionário aos juízes especialistas teve como objetivo a análise geral da avaliação, visando o julgamento do conteúdo, escopo da avaliação, linguagem, estrutura, clareza e objetividade. O primeiro questionário deve ser estruturado partindo de questões iniciais mais amplas e exploratórias, para aproximação dos participantes à temática estudada (AImeida et al., 2009).

Como resultado da $1^{\text {a }}$ rodada foi possível a caracterização do painel de juízes especialistas que corresponderam aos critérios da pesquisa. Participaram quatro terapeutas ocupacionais e quatro fonoaudiólogos, um participante com mestrado em andamento, três com mestrado concluído, três com doutorado em andamento e um com doutorado concluído. Em relação ao conhecimento e experiência, todos declararam ser conhecedores da Classificação Internacional de Funcionalidade, Incapacidade e Saúde - CIF. Cinco referiram a deficiência visual e reabilitação em deficiência visual. Quatro disseram ter conhecimento e experiência em avaliação da visão funcional e em Terapia Ocupacional. Três juízes declararam conhecimentos quanto ao processo de avaliação em Terapia Ocupacional e um em processos de validação e confiabilidade de instrumentos de avaliação.

A escolha dos juízes especialistas é fundamental e influencia diretamente na qualidade da pesquisa. Grupos heterogêneos, a partir de olhares variados, geram informações, sugestões e soluções heterogêneas e complementares, o que oportuniza e possibilita novas perspectivas à pesquisa (Grant \& Davis, 1997; Powell, 2003; Habibi et al., 2014; Revorêdo et al., 2015; Waggoner et al., 2016; Marques \& Freitas, 2018). De acordo com Marques e Freitas, "é importante que o painel seja equilibrado entre imparcialidade e interesse no assunto e que seja variado em termos de experiência, áreas de especialidade e perspectivas em relação ao problema" (2018, p.395).

O questionário da $1^{\text {a }}$ rodada da técnica Delphi foi composto por treze questões, através de duas modalidades diferentes e complementares de perguntas para a coleta das informações - resposta em cinco pontos, respostas dicotômicas e abertas. Na tabela 2, são apresentadas afirmações, para cada afirmação o participante deveria conferir sua opinião entre cinco possibilidades: discordo completamente, discordo parcialmente, nem concordo e nem discordo, concordo parcialmente e concordo completamente. Para o cálculo de índice de validade de conteúdo item, os dois últimos pontos devem ser considerados.

Após (tabela 3) foram apresentadas perguntas em que a resposta deveria ser classificada em dois pontos: "sim" e "não". Havia um espaço próprio para o participante dissertar sobre suas opiniões, tecer considerações e observações. O especialista foi estimulado constantemente a aprofundar suas respostas e, assim, auxiliar nas adequações da Avaliação Terapêutica Ocupacional (ATO) e na formulação de um novo questionário. Isso possibilitou chegar nas etapas seguintes do processo de validação, isto é, em novas rodadas da técnica Delphi.

O índice de validade de conteúdo total obtido foi de $0,892(89,2 \%)$ o que, até o momento, correspondeu a $\leq 0,90$ ou $90 \%$ estabelecido como índice de 
Tabela 2. Julgamento dos juízes especialistas sobre a $\mathrm{ATO}_{1}-1^{\mathrm{a}}$ rodada técnica Delphi - Avaliação em cinco pontos.

\begin{tabular}{|c|c|c|c|c|c|c|}
\hline \multirow[t]{2}{*}{ Afirmações } & \multicolumn{6}{|c|}{$\begin{array}{l}\text { Grau de concordância (n e IVC item \%) } \\
n=8\end{array}$} \\
\hline & DC & DP & NN & CP & CC & IVC item \\
\hline $\begin{array}{l}\text { 1. Na minha opinião, a } \\
\text { maneira como a ATO } \\
\text { está organizada é } \\
\text { adequada. }\end{array}$ & - & - & $\begin{array}{c}2 \\
25 \%\end{array}$ & $\begin{array}{c}1 \\
12,5 \%\end{array}$ & $\begin{array}{c}5 \\
62,5 \%\end{array}$ & 0,75 ou $75 \%$ \\
\hline $\begin{array}{l}\text { 2. Na minha opinião, a } \\
\text { ordem dos tópicos da } \\
\text { ATO está adequada. }\end{array}$ & - & - & $\begin{array}{c}1 \\
12,5 \%\end{array}$ & $\begin{array}{c}1 \\
12,5 \%\end{array}$ & $\begin{array}{c}6 \\
75 \%\end{array}$ & $\begin{array}{c}0,875 \text { ou } \\
87,5 \%\end{array}$ \\
\hline $\begin{array}{l}\text { 3. Na minha opinião, a } \\
\text { ATO está escrita com } \\
\text { linguagem acessível. }\end{array}$ & - & - & - & $\begin{array}{c}3 \\
37,5 \%\end{array}$ & $\begin{array}{c}5 \\
62,5 \%\end{array}$ & 0,1 ou $100 \%$ \\
\hline $\begin{array}{l}\text { 4. Na minha opinião, a } \\
\text { ATO está escrita de forma } \\
\text { clara. }\end{array}$ & - & - & - & $\begin{array}{c}2 \\
25 \%\end{array}$ & $\begin{array}{c}6 \\
75 \%\end{array}$ & 0,1 ou $100 \%$ \\
\hline $\begin{array}{l}\text { 5. Na minha opinião, a } \\
\text { ATO está escrita de forma } \\
\text { objetiva. }\end{array}$ & - & - & $\begin{array}{c}1 \\
12,5 \%\end{array}$ & $\begin{array}{c}1 \\
12,5 \%\end{array}$ & $\begin{array}{c}6 \\
75 \%\end{array}$ & $\begin{array}{c}0,875 \mathrm{ou} \\
87,5 \%\end{array}$ \\
\hline $\begin{array}{l}\text { 6. Na minha opinião, a } \\
\text { ATO apresenta } \\
\text { informações suficientes } \\
\text { para sua compreensão. }\end{array}$ & - & $\begin{array}{c}1 \\
12,5 \%\end{array}$ & $\begin{array}{c}1 \\
12,5 \%\end{array}$ & $\begin{array}{c}2 \\
25 \%\end{array}$ & $\begin{array}{c}4 \\
50 \%\end{array}$ & 0,75 ou $75 \%$ \\
\hline
\end{tabular}

Fonte: Silva (2020).

concordância mínimo para sua validade. Após a análise, nenhuma questão foi excluída da Avaliação. Entretanto, tornou-se compulsória a modificação da sequência dos itens (questão 7 - tabela 3), uma vez que obteve IVC item = $0,625(62,5 \%)$ favorável às alterações na Avaliação. Para outras quatro questões, o IVC item foi 0,75 (75\%) necessitando de revisão e adequação (questão 1, questão 6 - tabela 2; questão 11.4, questão 11.8 - tabela 3).
Destacaram-se algumas necessidades apontadas pelos juízes nas questões que receberam IVC item $\leq 0,78(78 \%)$, como:

- A construção de um manual que visasse a contextualização da Avaliação Terapêutica Ocupacional, seus objetivos, base teórica, métodos sugeridos de aplicação, preenchimento das informações e análise das respostas identificadas. Apesar da $\mathrm{ATO}_{1}$ ter instruções iniciais, nem todos os aspectos soli- 
Tabela 3. Julgamento dos juízes especialistas sobre a $\mathrm{ATO}_{1}-1^{\mathrm{a}}$ rodada técnica Delphi - Avaliação em dois pontos.

\begin{tabular}{|c|c|c|}
\hline \multirow[t]{2}{*}{ Questões } & \multicolumn{2}{|c|}{$\begin{array}{c}\text { Grau de } \\
\text { concordância } \\
\text { (n e IVC item \%) } \\
\mathbf{n = 8}\end{array}$} \\
\hline & Sim & Não \\
\hline $\begin{array}{l}\text { 7. Você mudaria a sequência dos itens da ATO? (sequência atual: } \\
\text { instruções gerais, dados pessoais, aspectos visuais, entrevista } \\
\text { semiestruturada, atividade prática, instruções e quadros para atividade } \\
\text { prática) }\end{array}$ & $\begin{array}{c}5 \\
62,5 \%\end{array}$ & $\begin{array}{c}3 \\
37,5 \%\end{array}$ \\
\hline $\begin{array}{l}\text { 8. A ATO possui extensão adequada, tendo em vista os aspectos que } \\
\text { pretende avaliar? }\end{array}$ & $\begin{array}{c}7 \\
87,5 \%\end{array}$ & $\begin{array}{c}1 \\
12,5 \%\end{array}$ \\
\hline 9. A ATO possui alguma palavra difícil de compreender seu significado? & - & $\begin{array}{c}8 \\
100 \%\end{array}$ \\
\hline $\begin{array}{l}\text { 10. A ATO possui alguma palavra que tenha duplo sentido ou dupla } \\
\text { interpretação? }\end{array}$ & - & $\begin{array}{c}8 \\
100 \% \\
\end{array}$ \\
\hline \multicolumn{3}{|l|}{ 11. Considero que a ATO contempla os aspectos: } \\
\hline 11.1 Aspectos visuais & $\begin{array}{c}8 \\
100 \% \\
\end{array}$ & - \\
\hline 11.2 Funcionalidade da pessoa com deficiência visual & $\begin{array}{c}8 \\
100 \%\end{array}$ & - \\
\hline 11.3 Dificuldades cotidianas da pessoa com deficiência visual & $\begin{array}{c}8 \\
100 \% \\
\end{array}$ & - \\
\hline 11.4 Restrições na participação da pessoa com deficiência visual & $\begin{array}{c}6 \\
75 \% \\
\end{array}$ & $\begin{array}{c}2 \\
25 \% \\
\end{array}$ \\
\hline $\begin{array}{l}\text { 11.5 Limitação na realização das atividades da pessoa com } \\
\text { deficiência visual }\end{array}$ & $\begin{array}{c}8 \\
100 \% \\
\end{array}$ & - \\
\hline 11.6 Capacidades da pessoa com deficiência visual & $\begin{array}{c}7 \\
87,5 \%\end{array}$ & $\begin{array}{c}1 \\
12,5 \%\end{array}$ \\
\hline 11.7 Interesses da pessoa com deficiência visual & $\begin{array}{c}7 \\
87,5 \% \\
\end{array}$ & $\begin{array}{c}1 \\
12,5 \% \\
\end{array}$ \\
\hline 11.8 Expectativas da pessoa com deficiência visual & $\begin{array}{c}6 \\
75 \% \\
\end{array}$ & $\begin{array}{c}2 \\
25 \% \\
\end{array}$ \\
\hline 11.9 Barreiras ambientais da pessoa com deficiência visual & $\begin{array}{c}8 \\
100 \%\end{array}$ & - \\
\hline 11.10 Facilitadores ambientais da pessoa com deficiência visual & $\begin{array}{c}7 \\
87,5 \%\end{array}$ & $\begin{array}{c}1 \\
12,5 \%\end{array}$ \\
\hline 12. A ATO, como um todo, mostrou-se viável de ser aplicada? & $\begin{array}{c}8 \\
100 \%\end{array}$ & - \\
\hline $\begin{array}{l}\text { 13. Na sua opinião, o nome da ATO é adequado? (Avaliação Terapêutica } \\
\text { Ocupacional para Adolescentes e Adultos com Deficiência Visual) }\end{array}$ & $\begin{array}{c}8 \\
100 \% \\
\end{array}$ & - \\
\hline
\end{tabular}

Fonte: Silva (2020). 
citados e sugeridos pelos juízes eram contemplados. Segundo alguns especialistas, a ausência de um manual dificulta a compreensão da avaliação, prejudicando, inclusive, sua clareza e aplicabilidade;

- Mudança da disposição das instruções de cada questão. Anteriormente, havia um quadro inicial com as instruções gerais de todos os itens da Avaliação reunidas. A partir das sugestões, houve a aproximação da instrução com seu respectivo item/ questão, no decorrer do corpo da Avaliação;

- Inclusão do item "relações íntimas";

- Necessidade de identificação das expectativas da pessoa com deficiência visual, tanto em relação à Avaliação Terapêutica Ocupacional, quanto ao processo de reabilitação que estaria iniciando e/ou participando.

Outras sugestões foram apontadas pelo painel de participantes que, embora não tenham impactado no valor de índice de validade de conteúdo item, representaram opiniões relevantes, como por exemplo, a inclusão de itens, como "religião" e "atividades domésticas"; reformulação do quadro sobre as atividades de vida diária.

A ATO ${ }_{1}$ possuía dois quadros. No primeiro, a pessoa com deficiência visual poderia referir dificuldades em realizar ou participar de determinadas atividades cotidianas. As atividades elencadas na Avaliação Terapêutica Ocupacional foram identificadas em pesquisas com pessoas com deficiência visual (Silva, 2016). No segundo quadro, era possível qualificar o nível de dificuldade das atividades identificadas como difíceis, através dos qualificadores da Classificação Internacional de Funcionalidade, Incapacidade e Saúde - CIF. A partir da modificação sugerida nessa rodada, os dois quadros foram unidos, tornando-os mais autoexplicativos e fáceis de preencher.

Todas as observações, críticas e sugestões foram analisadas pela pesquisadora. Foram incorporadas à Avaliação, parcial ou totalmente, as considerações que correspondessem à literatura utilizada como base teórica e que se mostraram relevantes ao tema proposto: uma avaliação dentro das competências do terapeuta ocupacional e do escopo da Classificação Internacional de Funcionalidade, Incapacidade e Saúde - CIF para pessoas com deficiência visual, adolescentes e adultos.

A partir do cálculo de índice de validade de conteúdo item, como resultado da $1^{\text {a }}$ rodada (tabelas 1,2 e 3 ), pelo julgamento dos juízes especialistas, a Avaliação apresenta linguagem acessível, escrita clara e objetiva, ordem dos tópicos e extensão adequadas. Não possui palavras difíceis de compreender o significado e nem palavras de duplo sentido e interpretação. Ainda segundo o painel, a Avaliação Terapêutica Ocupacional contempla os aspectos visuais, a funcionalidade, dificuldades cotidianas, limitações, capacidades, interesses, barreiras e facilitadores da pessoa com deficiência visual, mostrando-se viável de ser aplicada e com nome adequado ao que se propõe.

As adequações realizadas na $\mathrm{ATO}_{1}$ deram origem à $\mathrm{ATO}_{2}$ e ao seu manual de aplicação. Tais adequações foram julgadas na $2^{a}$ rodada da técnica Delphi. 


\section{$2^{a}$ rodada da técnica Delphi}

A $2^{\text {a }}$ etapa da técnica Delphi, para validação da Avaliação Terapêutica Ocupacional, deu-se a partir do envio da $\mathrm{ATO}_{2}$, e seu respectivo manual, aos juízes especialistas (tabela 1).

O questionário da $2^{\mathrm{a}}$ rodada teve sua origem nas modificações, sugestões e observações realizadas durante a $1^{\text {a }}$ etapa pelos juízes. Nesse momento da pesquisa, buscou-se, assim como aponta a literatura, o aprofundamento de perguntas e aspectos relacionados à Avaliação Terapêutica Ocupacional para que, ao final, fosse obtida uma avaliação validada (AImeida et al., 2009).

Todas as 17 questões da $2^{\mathrm{a}}$ rodada (tabela 4) possuíam espaço apropriado para expressão de comentários, sugestões e opiniões. Ao final, mais um espaço foi aberto para observações e sugestões - que não estavam contempladas nas perguntas realizadas. Os espaços que estavam em aberto, para que os participantes dissertassem, foram fundamentais para a análise qualitativa dos dados e para gerar mudanças na Avaliação (Kayo \& Securato, 1997). Os participantes eram constantemente estimulados a acrescentar comentários para auxiliar nas adequações da $\mathrm{ATO}_{2}$.

Como resultado da $2^{\mathrm{a}}$ rodada, nenhuma questão recebeu concordância IVC item $<0,75$ (75\%). Entretanto, uma questão recebeu IVC item $=0,75$ (75\%) (questão 2 - tabela 4), referente ao conteúdo e à extensão das informações sobre a Classificação Internacional de Funcionalidade, Incapacidade e Saúde - CIF dispostas no manual, sendo compulsoriamente revista. Dois participantes discordaram quanto à necessidade de inclusão do conteúdo da Classificação. Entretanto, apenas um deles expressou sua opinião. De acordo com o especialista, espera-se que o avaliador já tenha tal conhecimento ou, ao menos, que o busque na bibliografia de referência. Referiu que, havendo necessidade de o conteúdo permanecer no manual, deva estar mais sucinto e direcionar o terapeuta ocupacional para leituras complementares.

$\mathrm{Na} 2^{\mathrm{a}}$ rodada, apesar do IVC total = 0,941 (94,1\%) obtido, os juízes especialistas contribuíram com observações e sugestões importantes e relevantes à Avaliação. Tais comentários só foram possíveis com a presença dos espaços em aberto, dispostos no decorrer do questionário (Kayo \& Securato, 1997). Embora o valor de índice de validade de conteúdo total estivesse em conformidade com o valor padronizado, não houve estabilidade nas sugestões, o que não representou o consenso, não permitindo considerar a Avaliação válida.

Embora não tenham impactado nos valores de índices de validade de conteúdo item e total, alguns outros comentários e sugestões foram oferecidos pelos juízes, como:

- A diminuição do conteúdo do manual de uma forma geral, tornando-o mais conciso e objetivo;

- Adequação de questões referentes ao ambiente domiciliar, acrescentando questões mais amplas sobre as atividades nesse ambiente, incluindo a identificação de facilitadores e barreiras;

- Em relação ao aspecto da religião, adicionado a esta nova versão $\left(\mathrm{ATO}_{2}\right)$, a partir da sugestão de inclusão na ATO $_{1^{\prime}}$ houve apenas um questionamento, isto 
é, se haveria de fato relevância tal informação para a compreensão do papel dessa atividade na vida da pessoa com deficiência visual. Por tratar-se de uma questão importante, há a necessidade de justificá-la.

Tabela 4. Julgamento dos juízes especialistas sobre a $\mathrm{ATO}_{2}-2^{\mathrm{a}}$ rodada técnica Delphi - Avaliação em dois pontos.

\begin{tabular}{|c|c|c|}
\hline \multirow[t]{2}{*}{ Questões } & \multicolumn{2}{|c|}{$\begin{array}{l}\text { Grau de } \\
\text { concordância (n e } \\
\text { IVC item \%) } \\
n=8\end{array}$} \\
\hline & Sim & Não \\
\hline $\begin{array}{l}\text { 1. Construção de um manual com informações sobre objetivo da ATO, } \\
\text { estrutura da avaliação, métodos de aplicação e terminologias } \\
\text { empregadas. Você acredita que o manual construído possui informações } \\
\text { suficientes para a aplicação da ATO por terapeutas ocupacionais? }\end{array}$ & $\begin{array}{c}8 \\
100 \%\end{array}$ & - \\
\hline $\begin{array}{l}\text { 2. Inclusão de breve explicação sobre a CIF, seus objetivos, componentes } \\
\text { e qualificadores. Você acredita ser importante ter uma breve explicação } \\
\text { sobre a CIF no manual? }\end{array}$ & $\begin{array}{c}6 \\
75 \%\end{array}$ & $\begin{array}{c}2 \\
25 \%\end{array}$ \\
\hline $\begin{array}{l}\text { 3. Inclusão de breve explicação sobre a CIF, seus objetivos, componentes } \\
\text { e qualificadores. Você acredita que as informações oferecidas sobre a CIF } \\
\text { são suficientes para a aplicação da ATO? }\end{array}$ & $\begin{array}{c}8 \\
100 \%\end{array}$ & - \\
\hline $\begin{array}{l}\text { 4. As instruções específicas de cada questão foram colocadas } \\
\text { imediatamente antes da pergunta que Ihe diz respeito. Você concorda } \\
\text { com a nova disposição das instruções de cada pergunta? }\end{array}$ & $\begin{array}{c}7 \\
87,5 \%\end{array}$ & $\begin{array}{c}1 \\
12,5 \%\end{array}$ \\
\hline $\begin{array}{l}\text { 5. Adequação da numeração das tabelas e quadros; revisão dos números } \\
\text { das questões. Você concorda com essas alterações? }\end{array}$ & $\begin{array}{c}8 \\
100 \% \\
\end{array}$ & - \\
\hline $\begin{array}{l}\text { 6. Inclusão, no item dados pessoais, sobre religião. Você concorda com } \\
\text { o acréscimo dessa questão? }\end{array}$ & $\begin{array}{c}7 \\
87,5 \% \\
\end{array}$ & $\begin{array}{c}1 \\
12,5 \% \\
\end{array}$ \\
\hline $\begin{array}{l}\text { 7. Melhores descrições referentes à escolaridade e atividade profissional, } \\
\text { no item dados pessoais. Você concorda com essas alterações? }\end{array}$ & $\begin{array}{c}7 \\
87,5 \% \\
\end{array}$ & $\begin{array}{c}1 \\
12,5 \% \\
\end{array}$ \\
\hline $\begin{array}{l}\text { 8. Alteração e adequação da questão } 4.4 \text { "Dificuldades nas atividades } \\
\text { básicas e instrumentais de vida diária". Você concorda com a mudança } \\
\text { no quadro das atividades com a junção do quadro de magnitude da } \\
\text { dificuldade? }\end{array}$ & $\begin{array}{c}8 \\
100 \%\end{array}$ & - \\
\hline $\begin{array}{l}\text { 9. Inclusão de "relações íntimas" no quadro de atividades com possível } \\
\text { dificuldade (pergunta 4.4). Você concorda com a inclusão desta } \\
\text { atividade? }\end{array}$ & $\begin{array}{c}8 \\
100 \%\end{array}$ & - \\
\hline $\begin{array}{l}\text { 10. Na pergunta } 4.5 \text {, você concorda com o novo formato ao questionar } \\
\text { sobre as atividades prioritárias para o processo de reabilitação? }\end{array}$ & $\begin{array}{c}8 \\
100 \% \\
\end{array}$ & - \\
\hline $\begin{array}{l}\text { 11. Inclusão de perguntas gerais sobre atividades domésticas, na questão } \\
5 \text {. Você concorda com a inclusão de perguntas referentes às atividades e } \\
\text { ambiente doméstico? }\end{array}$ & $\begin{array}{c}8 \\
100 \%\end{array}$ & - \\
\hline 12. Sobre a questão 5.1., acredita que as questões feitas sejam relevantes? & $\begin{array}{c}7 \\
87,5 \% \\
\end{array}$ & $\begin{array}{c}1 \\
12,5 \% \\
\end{array}$ \\
\hline $\begin{array}{l}\text { 13. Inclusão e adequação de perguntas nas atividades profissionais, na } \\
\text { questão 5.3. Você concorda com tais alterações e adequações? }\end{array}$ & $\begin{array}{c}7 \\
87,5 \% \\
\end{array}$ & $\begin{array}{c}1 \\
12,5 \% \\
\end{array}$ \\
\hline $\begin{array}{l}\text { 14. Adequação e inclusão das instruções para aplicação da questão } 6 \\
\text { "relacionamento interpessoal". Você acredita que agora, com a presença } \\
\text { das instruções, a questão } 6 \text { ficou mais fácil de interpretar e perguntar ao } \\
\text { avaliado? }\end{array}$ & $\begin{array}{c}8 \\
100 \%\end{array}$ & - \\
\hline $\begin{array}{l}\text { 15. Na questão } 7 \text {, "aspecto socioemocional", houve a inclusão de } \\
\text { perguntas. Você concorda com o acréscimo? }\end{array}$ & $\begin{array}{c}8 \\
100 \% \\
\end{array}$ & - \\
\hline $\begin{array}{l}\text { 16. Inclusão de quadro final com a descrição dos códigos utilizados na } \\
\text { ATO. Você acredita ser importante ter o quadro ao final da ATO? }\end{array}$ & $\begin{array}{c}7 \\
87,5 \% \\
\end{array}$ & $\begin{array}{c}1 \\
12,5 \% \\
\end{array}$ \\
\hline $\begin{array}{l}\text { 17. Inclusão das receitas sugeridas para realização da atividade prática } \\
\text { de culinária. Você acredita ser importante as receitas constarem ao final } \\
\text { da ATO? }\end{array}$ & $\begin{array}{c}8 \\
100 \%\end{array}$ & - \\
\hline
\end{tabular}

Fonte: Silva (2020). 
Atividades de expressão religiosa e espiritual, para a Terapia Ocupacional, são consideradas atividades instrumentais de vida diária e, portanto, determinantes na vida dos sujeitos. A religião e a espiritualidade podem consistir em maneiras distintas de realizar a mesma atividade. Podem promover a participação social, também podem ser consideradas atividades de lazer ou mesmo profissionais. Podem levar à rituais específicos, vestimentas específicas, levar à hábitos. A religiosidade e a espiritualidade podem influenciar diretamente na maneira como a deficiência é percebida, vivida e encarada pessoal e socialmente. Além disso, pode ser tanto uma barreira como um fator facilitador para a pessoa com deficiência (AOTA, 2015).

A partir da análise pelo cálculo do índice de validade de conteúdo item, os participantes julgaram o manual com informações suficientes para a aplicação da Avaliação Terapêutica Ocupacional e aceitaram as adequações resultantes da $1^{\mathrm{a}}$ rodada.
Como resultado da $2^{\text {a }}$ rodada da técnica Delphi para validação, há a nova versão da Avaliação Terapêutica Ocupacional, chamada aqui de $\mathrm{ATO}_{3^{\prime}}$ com a separação estética do manual e da Avaliação propriamente dita. O conteúdo de seu manual é reduzido, mais objetivo, possibilita e estimula o profissional terapeuta ocupacional a consultar fontes originais na literatura, para seu aprofundamento teórico, além da adição e reorganização de itens.

\section{$3^{\mathrm{a}}$ rodada técnica Delphi}

A $3^{\mathrm{a}}$ rodada teve início com o envio da $\mathrm{ATO}_{3}$ aos juízes especialistas. Todas as perguntas do questionário (tabela 5) possuíam espaço apropriado para expressão de opiniões. Os participantes foram constantemente estimulados a acrescentar comentários para auxiliar nas adequações da $\mathrm{ATO}_{3}$.

Tabela 5. Julgamento dos juízes especialistas sobre a $\mathrm{ATO}_{3}-3^{\mathrm{a}}$ rodada técnica Delphi - Avaliação em dois pontos.

\begin{tabular}{|c|c|c|}
\hline \multirow[t]{2}{*}{ Questões } & \multicolumn{2}{|c|}{$\begin{array}{l}\text { Grau de } \\
\text { concordância (n e } \\
\text { IVC item \%) } \\
n=8\end{array}$} \\
\hline & Sim & Não \\
\hline $\begin{array}{l}\text { 1. O manual sofreu mudanças, resultando em um corpo de texto mais } \\
\text { sucinto. Você está de acordo com as alterações no manual, como um } \\
\text { todo? }\end{array}$ & $\begin{array}{c}8 \\
100 \%\end{array}$ & - \\
\hline $\begin{array}{l}\text { 2. O texto sobre a explicação da CIF sofreu alterações, passando a } \\
\text { apresentar-se com menos detalhes sobre sua aplicabilidade, estrutura e } \\
\text { códigos. O avaliador é convidado a consultar as referências da } \\
\text { Organização Mundial da Saúde sobre o assunto. Você concorda com a } \\
\text { alteração realizada? }\end{array}$ & $\begin{array}{c}8 \\
100 \%\end{array}$ & - \\
\hline $\begin{array}{l}\text { 3. No item "dados pessoais - escolaridade" acrescentou-se a informação } \\
\text { "pós-graduação". Você concorda com a inserção? }\end{array}$ & $\begin{array}{c}8 \\
100 \% \\
\end{array}$ & - \\
\hline $\begin{array}{l}\text { 4. As instruções de cada item passaram a ter destaque em quadros para } \\
\text { uma separação estética. Você concorda com a alteração e a nova } \\
\text { apresentação? }\end{array}$ & $\begin{array}{c}8 \\
100 \%\end{array}$ & - \\
\hline $\begin{array}{l}\text { 5. O item } 5.1 \text { "atividades domésticas" foi reorganizado e uma questão } \\
\text { foi adicionada. Você concorda com as alterações realizadas? }\end{array}$ & $\begin{array}{c}8 \\
100 \%\end{array}$ & - \\
\hline $\begin{array}{l}\text { 6. Após as alterações resultantes da } 1^{\mathrm{a}} \text { e } 2^{\mathrm{a}} \text { rodadas, você acredita que } \\
\text { a ATO esteja pronta para ser utilizada? }\end{array}$ & $\begin{array}{c}7 \\
87,5 \%\end{array}$ & $\begin{array}{c}1 \\
12,5 \%\end{array}$ \\
\hline
\end{tabular}

Fonte: Silva (2020) 
Nesta rodada da técnica Delphi não houve exclusão de nenhum item na Avaliação Terapêutica Ocupacional, bem como a necessidade compulsória de adequações. Não foram sugeridas alterações e oferecidas opiniões que provocassem uma nova rodada de questionário. Os comentários do painel de juízes foram positivos quanto às mudanças ocorridas durante o processo de validação.

Os participantes julgaram a Avaliação e seu manual como adequados, seja em seu conteúdo, estrutura, clareza, aplica- bilidade, linguagem e objetividade. Sendo considerada estável diante da ausência de novas sugestões de modificações e com o índice de validade de conteúdo total de 0,97 (97,91\%), a Avaliação Terapêutica Ocupacional (ATO) para Adolescentes e Adultos com Deficiência Visual passou a ser considerada válida. A tabela 6 ilustra a organização da Avaliação, após o processo de validação.

Destacamos que, apesar da literatura apontar para a tendência de perda de participantes em pesquisas que fazem

Tabela 6. Organização da Avaliação Terapêutica Ocupacional (ATO) para Adolescentes e Adultos com Deficiência Visual, após o processo de validação.

\begin{tabular}{|c|c|}
\hline \multicolumn{2}{|l|}{ Informações prévias } \\
\hline Manual da ATO & $\begin{array}{l}\text { Constam informações e orientações ao } \\
\text { avaliador para a aplicação da ATO, } \\
\text { interpretação dos resultados e conceitos } \\
\text { empregados. }\end{array}$ \\
\hline \multicolumn{2}{|l|}{ Avaliação propriamente dita } \\
\hline I. Identificação dos dados pessoais & Coleta de informações dos fatores pessoais. \\
\hline II. Informações dos aspectos visuais & $\begin{array}{l}\text { Inclui diagnóstico oftalmológico, preferência } \\
\text { de iluminação, uso de recursos ópticos, não } \\
\text { ópticos, cansaço visual, dados das funções } \\
\text { visuais. Em anexo, constam as avaliações das } \\
\text { funções visuais sugeridas. }\end{array}$ \\
\hline III. Entrevista semiestruturada & $\begin{array}{l}\text { Inclui informações das atividades cotidianas, } \\
\text { dificuldades nas AVDs e AIVDs, atividades } \\
\text { educacionais e profissionais, relacionamento } \\
\text { interpessoal, aspecto socioemocional. }\end{array}$ \\
\hline IV. Observação direta & $\begin{array}{l}\text { Realização de tarefa funcional e } \\
\text { preenchimento da lista mínima de } \\
\text { componentes da CIF para qualificação das } \\
\text { capacidades apresentadas. }\end{array}$ \\
\hline
\end{tabular}

Fonte: Silva (2020) 
uso da técnica Delphi, conforme novas rodadas são desenvolvidas, nesta pesquisa, os oito participantes permaneceram ativos durante todo o processo de validação. De acordo com Grisham (2009) e Marques e Freitas (2018), a seleção do painel deve ser cuidadosa, sendo características importantes o comprometimento e a disponibilidade em participar de múltiplas rodadas. Coutinho et al. (2013) e Waggoner et al. (2016), identificaram em suas pesquisas que a taxa de retorno e participação, em novas rodadas, eram inversamente proporcionais. Quanto mais rodadas eram desenvolvidas, menos especialistas mantinham a participação.

Outro fator que cabe destaque para reflexão possui relação com os espaços em aberto, disponíveis nos questionários. Como evidenciado, principalmente nos resultados da $2^{a}$ rodada, os especialistas contribuíram com sugestões e comentários importantes para a validação da Avaliação, que só foram possíveis com a abertura desses espaços para sua expressão. Caso contrário, a Avaliação Terapêutica Ocupacional poderia ser considerada válida quantitativamente na $2^{\text {a }}$ rodada da técnica. O aprimoramento da Avaliação e o aprofundamento das questões nas $2^{\mathrm{a}}$ e $3^{\mathrm{a}}$ rodadas só foram possíveis a partir da opinião dos especialistas. Dessa forma, apesar da literatura não apontar um padrão para a construção dos questionários (Faro, 1997; Kayo \& Securato, 1997; Marques \& Freitas, 2018), a presença de espaços para os comentários foi identificada como fundamental para o desenvolvimento da técnica Delphi (Kayo \& Securato, 1997).

A Avaliação Terapêutica Ocupacional possibilita a realização da entre- vista semiestruturada, que identifica os fatores relacionados ao desempenho da pessoa com deficiência visual, realizando e participando das atividades em seu ambiente habitual. Possibilita também a observação direta em avaliação, através da realização de tarefas funcionais em ambiente padrão, sendo possível a observação das capacidades da pessoa. Ao fazer uso dessas duas estratégias, a Avaliação apresenta-se potente para a identificação dos fatores ambientais, como influenciadores diretos na realização e participação de adolescentes e adultos com deficiência visual em atividades cotidianas. De acordo com a Classificação Internacional de Funcionalidade, Incapacidade e Saúde -CIF, "a lacuna entre a capacidade e o desempenho reflete a diferença entre os impactos dos ambientes habitual e uniforme, fornecendo, assim, uma orientação útil sobre o que pode ser feito ao ambiente do indivíduo para melhorar seu desempenho" (OMS, 2015, p.268).

Sendo assim, ao utilizar a Avaliação Terapêutica Ocupacional, o terapeuta aproxima-se do relato da pessoa avaliada sobre os aspectos que, do ponto de vista do indivíduo, influenciam no seu cotidiano, são importantes e significativos. Também consegue observar as capacidades e as dificuldades em setting terapêutico, qualificar a magnitude da dificuldade apresentada e identificar demandas terapêuticas não verbalizadas pelo avaliado.

Finalmente, a Avaliação propõe, ao dialogar com a Classificação Internacional de Funcionalidade, Incapacidade e Saúde - CIF, o deslocamento do cuidado e do olhar terapêutico ocupacional sobre saúde, funcionalidade e deficiência como aspectos isolados. Considera 
a deficiência como resultado do corpo, com suas estruturas e funções, interagindo com fatores pessoais e externos, contextuais e ambientais, de natureza individual, familiar, histórica, social e cultural. Todos esses aspectos interferem diretamente na funcionalidade da pessoa, que experiência de forma particular sua vida e permite, assim, um fazer singularizado.

\section{Conclusões}

Este manuscrito descreveu o processo de validação da Avaliação Terapêutica Ocupacional (ATO) para Adolescentes e Adultos com Deficiência Visual, por meio de um painel de especialistas.

A técnica Delphi prevê um período ampliado de coleta de dados. Entretanto, o tempo para realização da validação, do convite à análise da última rodada, que ocorreu durante doze meses ininterruptos, é identificado como uma limitação. Embora não tenha levado a perda de especialistas nesta pesquisa, esse pode ser um fator determinante para a manutenção dos participantes, pois pode prejudicar o processo de validação, caso a diminuição de especialistas, entre as rodadas, seja significativa. Desta forma, a identificação de participantes em potencial, assim como já referido anteriormente neste manuscrito, deve ser cuidadosa.

Após três rodadas da técnica Delphi, a Avaliação Terapêutica Ocupacional é considerada válida em face e conteúdo, sob o julgamento de especialistas, podendo, agora, ser utilizada por terapeutas ocupacionais brasileiros. É um instrumento de avaliação que avalia o que se propõe, possui linguagem acessível, estrutura e conteúdo relevantes, claros, objetivos, adequados para atingir seu objetivo máximo: avaliar a funcionalidade de adolescentes e adultos com deficiência visual, por meio dos métodos de entrevista e observação direta. Identifica as principais ocupações e atividades cotidianas, limitações na realização e participação em atividades, faz o rastreio de facilitadores e barreiras ambientais e a observação das capacidades por meio de tarefa funcional. Além disso, aproxima o profissional do conhecimento da realidade da pessoa com deficiência visual a partir do olhar do avaliado, firmando o protagonismo no próprio processo de reabilitação.

A Avaliação Terapêutica Ocupacional padroniza a avaliação em Terapia Ocupacional para adolescentes e adultos com deficiência visual. Utiliza linguagem universal através da Classificação Internacional de Funcionalidade, Incapacidade e Saúde -CIF, o que facilita o diálogo entre terapeutas ocupacionais e entre esses e outros profissionais da rede de cuidado. Viabiliza a avaliação e a reavaliação, o que contribui para a análise das intervenções.

Por fim, após amplas pesquisas, será possível a formação de banco de dados sistematizado, com informações essenciais para compreender a realidade de adolescentes e adultos com deficiência visual, permitindo com isso o planejamento e a implementação de ações e políticas públicas que garantam a plena realização e participação em atividades cotidianas. 


\section{Referencias}

Alexandre, N. M. C. \& Coluci, M. Z. O. (2011). Validade de conteúdo nos processos de construção e adaptação de instrumentos de medidas. Ciência \& Saúde Coletiva, 16(7),3061-3068. http://dx.doi. org/10.1590/S1413-81232011000800006

Almeida, M. H. M. (2003). Validação do instrumento CICAc: Classificação de Idosos quanto à Capacidade para o Autocuidado [Tese de doutorado]. Faculdade de Saúde Pública, Universidade de São Paulo.

Almeida, M. H. M. (2004). Elaboração e validação do instrumento CICAc: classificação de idosos quanto à capacidade para o autocuidado. Revista de Terapia Ocupacional da Universidade de São Paulo, 15(3), 112-20. https://doi.org/10.11606/issn.2238-6149. v15i3p112-120

Almeida, M. H. M., Spínola, A. W. P, Iwamizu, P. S., Okura, R. I. S, Barroso, L. P. \& Lima, A. C. P. (2008). Confiabilidade do Instrumento para Classificação de Idosos quanto à Capacidade para o Autocuidado. Revista de Saúde Pública, 42(2), 317-323. https://doi.org/10.1590/S0034$\underline{89102008005000003}$

Almeida, M. H. M., Spínola, A. W. P \& Lancman, S. (2009). Técnica Delphi: validação de um instrumento para uso do terapeuta ocupacional em gerontologia. Revista de Terapia Ocupacional da Universidade de São Paulo, 20(1), 49-58. https://doi.org/10.11606/issn.2238-6149. v20i1p49-58
American Occupational Therapy Association [AOTA] (2015). Estrutura da prática da Terapia Ocupacional: domínio \& processo $3^{a}$ ed traduzida. Revista de Terapia Ocupacional da Universidade de São Paulo, 26(esp), 1-49. https://doi.org/10.11606/ issn.2238-6149.v26iespp1-49

Coutinho, S. S., Freitas, M. A., Pereira, M. J. B., Veiga, T. B., Ferreira, M. \& Mishima, S. M. (2013). O uso da técnica Delphi na pesquisa em atenção primária à saúde: revisão integrativa. Revista Baiana Saúde Pública, 37(3), 582-596. https:// doi.org/10.22278/2318-2660.2013.v37. n3.a398

Cruz, D. M. C. (2018). Os modelos de Terapia Ocupacional e as possibilidades para a prática e pesquisa no Brasil. Revista Interinstitucional Brasileira de Terapia Ocupacional, 2(3), 504-517. https:// revistas.ufrj.br/index.php/ribto/article/ view/18436

De Carlo, M. M. R. P. \& Bartalotti, C. C. (2001). Caminhos da Terapia Ocupacional. Em M. M. R. P. De Carlo \& C. C. Bartalotti (orgs.), Terapia ocupacional no Brasil: fundamentos e perspectivas (pp.19-40). Plexus Editora.

Dickie, V. (2011). O que é Ocupação? Em E. B. Crepeau, E. S. Cohn e B. A. B. Schell, Terapia Ocupacional: Willard \& Spackman (pp. 66-74). Guanabara Koogan.

Faro, A. C. M. (1997). Técnica Delphi na validação das intervenções de enfermagem. RevistadaEscolade Enfermagem daUSP, (31), 259-273. https://doi.org/10.1590/ $\underline{\text { S0080-62341997000200008 }}$ 
Gil, A. C. (2008). Métodos e técnicas de pesquisa social. Atlas.

Grant, J. S. \& Davis, L. L. (1997). Selection and use of content experts for instrument development. Research in Nursing \& Health, 20, 269-274. https://doi.org/10.1002/ (SICI)1098-240X(199706)20:3\%3C269::AID-NUR9\%3E3.0.CO;2-G

Grisham T. (2009). The Delphi technique: a method for testing complex and multifaceted topics. International Journal of Managing Projects in Business, 2(1),112-130. https:// doi.org/10.1108/17538370910930545

Habibi, A., Sarafrazi, A. \& Izadyar, S. (2014). Delphi technique theoretical framework in qualitative research. The International Journal Of Engineering And Science, 3(4), 08-13.

Kayo, E. K. \& Securato, J. R. (1997). Método Delphi: fundamentos, críticas e vieses. Cadernos de Pesquisa em Administração, 1(4), 51-61.

Lynn, M. R. (1986). Determination and quantification of content validity. Nursing Research, 35(6),382-5. https://doi. org/10.1097/00006199-198611000$\underline{00017}$

Marques, J. B. V. \& Freitas, D. (2018). Método Delphi: caracterização e potencialidades na pesquisa em Educação. Pro-Posições. 29(2), 389-415. https://doi. org/10.1590/1980-6248-2015-0140

Martins, G. A. (2006). Sobre confiabilidade e validade. Revista Brasileira de Gestão de Negócios, 8(20), 1-12. e: http:// www.spell.org.br/documentos/ver/6471/ sobre-confiabilidade-e-validade/i/pt-br
Massaroli, A., Martini, J. G., Lino, M. M., Spenassato, D. \& Massaroli, R. (2017). Método Delphi como referencial metodológico para a pesquisa em enfermagem. Texto \& Contexto - Enfermagem, 26(4), 1-9. https://doi.org/10.1590/0104$\underline{07072017001110017}$

Medeiros, M. H. R. (2010). Terapia Ocupacional: um enfoque epistemológico e social. EdUFSCAR.

Organização Mundial de Saúde [OMS] (2015). CIF: Classificação Internacional de Funcionalidade, Incapacidade e Saúde. Trad. Centro Colaborador da Organização Mundial da Saúde para a Família de Classificações Internacionais. EDUSP.

Polit, D. F. \& Beck, C. T. (2006). The content validity index: are you sure you know what's being reported? Critique and recomendations. Research in Nursing \& Health, (29),489-497. https://doi. org/10.1002/nur.20147

Powell, C. (2003). The Delphi technique: myths and realities. Journal of advanced nursing, 41(4),376-382. https://doi. org/10.1046/j.1365-2648.2003.02537.x

Revorêdo, L. S., Maia, R. S., Torres, G. V. \& Maia, E. M. C. (2015). O uso da técnica Delphi em saúde: uma revisão integrativa de estudos brasileiros. Arquivos de Ciências da Saúde, 22(2),16-21. https://doi. org/10.17696/2318-3691.22.2.2015.136

Silva, M. R. (2016). Avaliação terapêutica ocupacional para adolescentes e adultos com deficiência visual baseada na Classificação Internacional de Funcionalidade, Incapacidade e Saúde (CIF) [Dissertação de mestrado, Universidade Estadual de Campinas]. Repositório 
Unicamp. http://repositorio.unicamp.br/ handle/REPOSIP/321851

Silva, M. R. (2020). Validação da Avaliação Terapêutica Ocupacional (ATO) para Adolescentes e Adultos com Deficiência Visual [Tese de doutorado a ser publicada]. Programa de Pós-Graduação em Saúde, Interdisciplinaridade e Reabilitação, Universidade Estadual de Campinas -UNICAMP.

Souza, A. C., Alexandre, N. M. C. \& GuirardeIlo, E. B. (2017). Propriedades psicométricas na avaliação de instrumentos: avaliação da confiabilidade e da validade. Epidemiologia e Serviços De Saúde, 26(3), 649-659. https://doi.org/10.5123/ S1679-49742017000300022

Waggoner, J., Carline, J. \& Durning, S. J. (2016). Is there a consensus on consensus methodology? Descriptions and recommendations for future consensus research. Academic Medicine, 91(5),663-668. https://doi.org/10.1097/ ACM.0000000000001092 